\title{
Ação educativa sobre o rastreamento precoce do câncer de colo de útero: relato de experiência
}

\author{
Educational action on early screening for cervical cancer: experience report \\ Acción educativa sobre el cribado precoz del cáncer de cuello uterino: un informe de experiencia
}

Recebido: 14/11/2021 | Revisado: 20/11/2021 | Aceito: 25/12/2021 | Publicado: 05/01/2022

Larissa Duarte Miranda

ORCID: https://orcid.org/0000-0003-0597-4974 Centro Universitário Metropolitano da Amazônia, Brasil

E-mail: larissaduartemiranda01906@gmail.com

Gabriela Marsola Sandim

ORCID: https://orcid.org/0000-0001-8629-7172 Centro Universitário Metropolitano da Amazônia, Brasil

E-mail: gabiisandim@gmail.com

Larissa Freitas Santos

ORCID: https://orcid.org/0000-0003-0031-4950 Centro Universitário Metropolitano da Amazônia, Brasil E-mail: larissafreitas062@gmail.com

Manuela Evangelista Moraes

ORCID: https://orcid.org/0000-0002-1744-683X Centro Universitário Metropolitano da Amazônia, Brasil E-mail: manuelamorais54@gmail.com

Maria do Socorro da Cruz

ORCID: https://orcid.org/0000-0001-7438-0614 Centro Universitário Metropolitano da Amazônia, Brasil E-mail: cruzsocorro193@gmail.com

Lorrane Santos Silva

ORCID: https://orcid.org/0000-0001-5348-1424 Centro Universitário Metropolitano da Amazônia, Brasil E-mail: lorranegod12345678@gmail.com

Rubenilson Caldas Valois

ORCID: https://orcid.org/0000-0001-9120-7741 Centro Universitário Metropolitano da Amazônia, Brasil E-mail: rubenilsonvalois@gmail.com

\begin{abstract}
Resumo
Objetivo: relatar experiência de acadêmicas do curso de enfermagem ao participarem de uma ação educativa, realizando atividades educativas em saúde com mulheres de 25 a 64 anos, na ESF Mangueirão a respeito do rastreamento precoce do câncer de colo de útero, o grupo participante foi composto por 9 mulheres. Método: trata-se de um estudo quantitativo, descritivo, do tipo relato de experiência. Resultado: A ação foi desenvolvida após o levantamento de dados sobre o referido assunto, mediante as estratégias de explicar de forma concisa a temática, para o desenvolvimento da ação foi utilizado um instrumento educativo em forma de banner com informações pertinentes sobre o tema e uma dinâmica interativa de perguntas e respostas com auxílio de placas com sinais positivo e negativo sinalizando evidentemente a compreensão das participantes sobre o assunto abordado. Conclusão: desta forma através da atividade educativa realizada obtivemos grande participação do público-alvo com levantamento de dúvidas sobre o câncer de colo do útero, exame de Papanicolau e também questões relacionadas à sexualidade e ao corpo feminino. A atividade ocorreu de forma expositivo-participativa e de grande aprendizado pela equipe de acadêmicos como para a população-alvo.
\end{abstract}

Palavras-chave: Câncer do colo do útero; Saúde da mulher; Exame ginecológico.

\begin{abstract}
Objective: to report the experience of nursing students participating in an educational action, carrying out health educational activities with women aged 25 to 64 years, at the ESF Mangueirão regarding early screening for cervical cancer, the participating group was composed by 9 women. Method: this is a quantitative, descriptive study, of the experience report type. Result: The action was developed after collecting data on the subject, through the strategies of concisely explaining the theme, for the development of the action an educational instrument was developed in the form of a banner with important information on the subject and a dynamic interactive questions and answers with the help of signs with positive and negative signs, evidently signaling the participants' understanding of the topic addressed. Conclusion: in this way, through the educational activity carried out, we obtained great participation from
\end{abstract}


the target audience, raising doubts about cervical cancer, Pap smears and also issues related to sexuality and the female body. The activity takes place in an expository/participatory way, with great learning by the academic team as well as for a target population.

Keywords: Cervical cancer; Women's health; Gynecological examination.

\section{Resumen}

Objetivo: reportar la experiencia de estudiantes de enfermería que participaron en una acción educativa, realizando actividades educativas en salud con mujeres de 25 a 64 años, en la ESF Mangueirão en materia de detección precoz de cáncer de cuello uterino, el grupo participante estuvo compuesto por 9 mujeres. Método: se trata de un estudio cuantitativo, descriptivo, del tipo relato de experiencia. Resultado: La acción se desarrolló luego de recolectar datos sobre el tema, a través de las estrategias de explicar de manera concisa el tema, para el desarrollo de la acción se desarrolló un instrumento educativo en forma de banner con información importante sobre el tema y una dinámica interactiva de preguntas. y respuestas con la ayuda de signos con signos positivos y negativos, lo que evidentemente indica la comprensión de los participantes sobre el tema abordado. Conclusión: de esta manera, a través de la actividad educativa realizada, logramos una gran participación del público objetivo, generando dudas sobre el cáncer de cuello uterino, el Papanicolaou y también temas relacionados con la sexualidad y el cuerpo femenino. La actividad se desarrolla de forma expositiva / participativa, con gran aprendizaje tanto del equipo académico como de la población objetivo.

Palabras clave: Cáncer de cuello uterino; Salud de la mujer; Exploración ginecológica.

\section{Introdução}

O Câncer de colo de útero (CCU) é o terceiro tipo de tumor maligno com a maior incidência de acometimento em mulheres no Brasil e de outros países em desenvolvimento. A replicação viral ocorre no epitélio de revestimento do útero, causando comprometimentos dos tecidos subjacentes (OMS, 2018). Além deste, há o predomínio do câncer de mama e de pele, onde é um problema voltado à saúde da mulher (Silva et al., 2015).

Sabe-se que a detecção de forma precoce mediante ao exame de preventivo ou Papanicolau é o meio mais eficaz na detecção primária de possíveis lesões causadoras do câncer no colo de útero, havendo também a identificação de fatores secundários relacionados ao Papiloma vírus humana (HPV) (Rodrigues et al., 2013), que acomete mulheres acima de 25 anos ativas sexualmente, sendo um fator contribuinte para o câncer que ainda dizima diversas mulheres no mundo (INCA, 2020; Messias, 2018).

A infecção pelo Papiloma vírus humana (HPV) é o principal fator associado ao câncer do colo de útero (Oliveira et al., 2016). Entretanto, outras causas e fatores também podem estar relacionados, como o início da vida sexual precoce, faixa etária entre 25 a 59 anos, atividade sexual com múltiplos parceiros, herança genética, infecções pelo HIV, entre outros (Osis; Duarte, 2014).

Durante as fases iniciais, podem-se desenvolver sintomas e evoluir para os sangramentos vaginais que acontecem após a relação sexual, dores abdominais intensas ou, até mesmo, queixas urinárias (Sá et al., 2015).

Desse modo, é válido enfatizar o exame ginecológico PCCU (Preventivo do câncer do colo de útero), conhecido também como exame de preventivo, sendo este, um procedimento onde se realiza o rastreamento de células sugestivas de préinvasão ou lesões malignas no colo do útero, além de diagnosticar doenças de caráter infeccioso (Toninato, 2016).

É um método considerado de baixo custo e de fácil manuseio, apesar disto, o CCU ainda é um sério problema voltado à saúde pública que atinge mulheres de todas as classes e regiões da sociedade brasileira devido ao não conhecimento da importância de realizar o exame de preventivo e, também do possível diagnóstico, causando sérios transtornos ao avanço científico voltado à ginecologia, onde regride a ideia de tratamento precoce da população feminina (Leite et al.,2014).

O estudo tem como objetivo relatar a experiência adquirida em uma ação educativa, onde foi abordada a temática de forma ilustrativa com auxílio de um banner sobre "Rastreamento precoce do câncer de colo de útero", com mulheres que realizam acompanhamento em uma estratégia de saúde da família - ESF do município de Belém/PA. Além de, oferecer 
vivências oportunas para os acadêmicos na área profissional, além de orientar e trazer conhecimento para mulheres entre 25 e 64 sobre a prevenção precoce do câncer no colo do útero.

\section{Metodologia}

O presente estudo trata-se de relato de experiência do tipo qualitativo descritivo, baseado em realização de ações educativas, no qual abordam experiências vivenciadas por acadêmicos de enfermagem, onde buscam reflexões sobre os fenômenos sociais em estudo, fazendo coleta de dados, por meio de atividades educacionais e entrevistas com questões abertas, objetivando minimizar as várias problemáticas voltadas à saúde ginecológica (Carvalho \& Montenegro.,2012). Sobre isto, é válido ressaltar que o projeto foi realizado por 6 discentes do curso de graduação em enfermagem do Centro Universitário Metropolitano da Amazônia (UNIFAMAZ), no município de Belém/PA. O local escolhido foi a Estratégia Saúde da família (ESF) localizada no bairro mangueirão no município de Belém/Pa, onde o prosseguimento da ação se deu na sala de espera da unidade em uma sexta-feira dia 05 de Novembro de 2021 no período da manhã, sendo o dia agendado pela enfermeira responsável para realização do exame preventivo, onde se tem a maior demanda de mulheres entre 25 e 64 anos na unidade, estavam presentes cerca de nove mulheres nessa faixa etária participando de prática educacional devido ser a idade recomendada para a prevenção do câncer no colo do útero.

\section{Relato da Experiência e Discussão}

Após o levantamento de dados bibliográficos sobre o referido assunto e definido o dia, a ação foi desenvolvida em dois momentos, a primeira etapa foi através da utilização de instrumento educativo em forma de banner com informações pertinentes sobre o que é câncer no colo do útero; as formas de prevenção; tratamento; fatores de risco; os sinais e sintomas possíveis e a descrição da técnica do exame Papanicolau. Na segunda etapa utilizou-se como método uma atividade interativa de perguntas e respostas com placas que sinalizavam a compreensão do assunto abordado.

Para o desenvolvimento da atividade educativa, foram abordadas as principais características do exame Papanicolau e sua importância na prevenção do câncer do colo de útero, assim como a apresentação do material utilizado para a realização da ação, o banner como demonstração contendo as informações dos sinais e sintomas, e medidas profiláticas a respeito do assunto, Seguida das perguntas, e plaquinhas para a simulação dinâmica.

Durante a apresentação do banner a equipe realizadora da ação explicou a todas as mulheres presentes que o câncer pode ocorrer por pequenas lesões no colo uterino, modificações celulares, sendo o mais comum à infecção por algum tipo de Papiloma vírus humano (HPV) que é uma infecção sexualmente transmissível, por isso, a importância do exame para deteç̧ão precoce e o uso de preservativos para evitar contaminação, além de, esclarecer que a detecção precoce é importante para o tratamento, após isto, foi fornecido explicações sobre: idade para realizar o primeiro exame preventivo; mulheres com vida sexual ativa devem realizar o exame periodicamente; problemas decorrentes da não realização do exame, fatores de risco para o desenvolvimento do câncer como tabagismo, iniciação sexual precoce, multiplicidade de parceiros, história familiar e a predisposição genética; contaminação pelo Papiloma vírus humana (HPV), dentre outros conteúdos associados à temática.

A atividade de interação teve o objetivo de avaliar a compreensão das mulheres sobre o assunto, identificar possíveis dúvidas e responder às perguntas das participantes, os objetos utilizados para a dinâmica foram placas com sinais de positivo e negativo, sinalizando a compreensão de todas ao assunto abordado. Foram selecionadas algumas perguntas importantes como: na sua família tem histórico de câncer no colo do útero?; o exame deve ser realizado anualmente?; mulheres que já iniciaram a vida sexual devem começar a realizar o exame?; uso do preservativo é importante para prevenir o câncer?; conhecem a vacina do HPV ou já tomaram quando adolescente?; relação sexual e menstruação no dia do exame é indicado?; é normal sangrar e 
sentir cólicas depois de realizar o exame?, todas essas perguntas foram devidamente respondidas pelas acadêmicas realizadoras da ação.

O método de rastreamento do câncer de colo do útero no Brasil é o exame citopatológico, onde a coleta do material é indolor e de baixo custo (Ferreira et al., 2012). Porém, como preconiza o Instituto Nacional do Câncer (INCA), caso realizado de forma periódica há maior probabilidade que assegura a saúde feminina, ou seja, quando há irregularidade no tratamento ginecológico é importante que os profissionais de saúde criem estratégias para orientar sobre a necessidade de atualização dos resultados e a forma adequada de realização de exames a cada três anos, após dois exames normais realizados no intervalo de um ano. A repetição de um ano após o primeiro teste visa à possibilidade de falsos negativos em uma primeira rodada e a posterior periodicidade de três anos atende uma recomendação da Organização Mundial da Saúde (OMS), (Ferreira al., 2012).

O rastreio de CCU na sociedade brasileira é por meio de exame Preventivo do Colo de Útero (PCCU), que é oferecido de forma gratuita no Sistema Público de Saúde (SUS) que é realizado na Atenção Primária de Saúde (APS) direcionada as mulheres a partir de 25 anos e que já tiveram atividade sexual, onde o exame é coletado do material do colo uterino do meio ectocérvice e do meio endocérvice, onde a amostra é levada para laboratório é um exame considerado simples, porém, há resistência na realização desse exame devido ao medo do possível diagnóstico ou pelo constrangimento de realiza o exame por um enfermeiro do sexo masculino (dos Santos et al, 2015; INCA, 2016; Barbosa et al. 2018).

Percebe-se a real necessidade que as mulheres têm a respeito de informações tão importantes a cerca de um assunto tão imprescindível, o que corrobora com o material publicado pelo Ministério da Saúde acerca do assunto o qual relata que o câncer do colo de útero atinge principalmente as mulheres com maior dificuldade de acesso aos serviços de saúde (Brasil, 2016).

De acordo com Cecília Rotelli, presidente da Comissão Nacional Especializada em Vacinas da Federação Brasileira das Associações de Ginecologia e Obstetrícia (Febrasgo) há uma dificuldade de alcançar as pessoas desejadas com relação à vacinação chegando em torno de $90 \%$ e a maior dificuldade além da falta de acesso às informações é convencer os adolescentes a tomarem as doses necessárias (Tadeu, 2021).

A falta de informação muitas vezes está associada com a renda familiar, em uma pesquisa realizada por Melo et al. (2019), evidenciou-se que mulheres com rendas menores apresentaram uma associação bem inferior ao conhecimento do que as com renda mais alta.

A ausência ou a baixa procura do exame preventivo de colo de útero não é apenas uma realidade local, mas global, as mulheres têm pouco conhecimento sobre o assunto relacionado a importância do diagnóstico precoce, do exame de prevenção, dos sintomas, cuidados, entre outros (Melo et al.,2019).

Algumas mulheres embora tenham pouco conhecimento, buscam informações ou se previnem como podem, as que não buscam prevenção tem como motivos principais a falta de interesse, sentimento de vergonha, a falta de tempo e de parceiro, não gostar do exame ou tem medo (Melo et al., 2019). Nesse cenário, é de suma importância a atuação de equipe multidisciplinar em unidades de saúde pública responsável por realização de exames de preventivo, onde os profissionais da saúde em especial a enfermagem são os principais agentes realizador desse exame (de Sousa., 2021; da Costa et al., 2017).

O papel dos enfermeiros e de suas ações nas unidades de saúde é fundamental para divulgação de temáticas necessárias como essas, segundo a Organização Pan-americana de Saúde (OPAS), as ações estabelecidas e executadas por esses profissionais são extremamente eficientes para a sociedade, pois levam conhecimentos à população, na maioria das vezes aqueles sem acesso aos meios adequados se tornando muitas vezes o único recurso que essas pessoas possuem para obterem informações (Gaspar, 2021). 


\section{Considerações Finais}

A execução da atividade acadêmica propiciou ao grupo um acréscimo na formação, demonstrando sobremaneira que, dentre as diversas atividades inerentes à profissão de enfermagem, encontra-se as atividades educativas, onde a Identificação precoce do conhecimento sobre o câncer de colo uterino, assim como o exame preventivo auxiliam na mediação do cuidado entre o profissional e o seu cliente. A partir disto, deve-se fomentar conhecimento sobre esta temática, contribuindo deste modo com cuidados à saúde da mulher. Essa atividade permitiu agregar informações que foram repassadas para um grupo de mulheres, as quais, nem sempre tem um momento de expressar e conhecer de maneira correta seu corpo e sua saúde, mas também permitiu ao grupo a prática de ensino/aprendizagem e mediou o esclarecimento de dúvidas que se encontram no dia a dia da mulher, influenciando negativamente em hábitos saudáveis. Ademais, experiências positivas realizadas ao longo da formação acadêmica são de suma importância para estudos avançados sobre temas relevantes, fortalecendo a prática profissional, agregando ações construtivas e desenvolvidas pelo serviço.

\section{Referências}

Barbosa, A. P., Ricacheneisky, L. F., \& Daudt, C. (2018). Prevenção e rastreamento de neoplasias femininas: mama e colo do útero. Acta Méd, 39(2), 335-45.

Brasil. Instituto Nacional de Câncer José Alencar Gomes da Silva. (2016). Coordenação de Prevenção e Vigilância. Divisão de Detecção Precoce e Apoio à Organização de Rede. Diretrizes brasileiras para o rastreamento do câncer do colo do útero.

Carvalho, B. G. C. \& Montenegro, L. C. (2012). Metodologias de comunicação no processo de educação em saúde. R. Enferm. Cent. O. Min. 2012; 2(2): 27987. http://www.seer.ufsj.edu.br/index.php/recom/article/view/ 148/31612- Silva, P. V., Araújo, A. \& Araújo, M. R. N. Análise

da Costa, F. K. M., Weigert, S. P., Burci, L., \& do Nascimento, K. F. (2017). Os desafios do enfermeiro perante a prevenção do câncer do colo do útero.

de Sousa Silva, T. R., dos Santos, J. C. M., de Oliveira, J. S., Abreu, V. P. L., da Silva, R. R., Dantas, K. L. S., \& Ferreira, R. K. A. (2021). A importância do exame Preventivo de Câncer de Colo de Útero e os fatores relacionados a não adesão. Research, Society and Development, 10(4), e51710414079e51710414079.

dos Santos Silva, M. A., Teixeira, É. M. B., Ferrari, R. A. P., Cestari, M. E. W., \& Cardelli, A. A. M. (2015). Fatores relacionados a não adesão à realização do exame de Papanicolau. Rev Rene, 16(4), 532-539.

Ferreira, L. C. \& Vogt, M. A. (2012). Exame De Papanicolau: Conhecimento de Usuárias De uma Unidade Básica de Saúde. Rev Enferm UFSM. 2(3):619-29

Gaspar, Izabelle. A importância da enfermagem na prevenção do câncer de colo de útero. PEBMED,2021. https://pebmed.com.br/a-importancia-daenfermagem-na-prevencao-do-cancer-de-colo-de-utero/.

INCA (2020). Instituto Nacional de Câncer José Alencar Gomes da Silva. Colo do útero. https://www.inca.gov.br/tipos-de-cancer/cancer-do-colo-do-utero

Leite, M. F., et al. Knowledge and practice of women regarding cervical cancer in a primary health care unit, Journal of Human Growth and Development, 24(2): 208-213 Manuscript submitted Oct 08 2013, accepted for publication Feb 222014.

Melo, E. M. F. D., Linhares, F. M. P., Silva, T. M. D., Pontes, C. M., Santos, A. H. D. S., \& Oliveira, S. C. D. (2019). Câncer cervico-uterino: conhecimento, atitude e prática sobre o exame de prevenção. Revista Brasileira de Enfermagem, 72, 25-31.

Messias, A. C. C. (2018). Prevenção do câncer do colo do útero: Desafios e perspectivas para a vacinação contra o hpv na região de saúde noroeste do Estado do Rio de Janeiro. Acta Biomédica Brasiliensia, 9(2), 42-57.

Ministério da Saúde. Instituto Nacional do Câncer - INCA INCA; 1996- 2018 HPV e câncer - Perguntas mais frequentes. https://www.inca.gov.br/perguntasfrequentes/hpv.

Ministério da Saúde. Instituto Nacional do Câncer - INCA INCA; 1996- 2018. HPV e câncer - Perguntas mais frequente; https://www.inca.gov.br/perguntasfrequentes/hpv

Oliveira, A. R. N., Nóbrega, M. M., Caldas, M. L. S. \& Nobre, J. O. C. (2016). Conhecimento das gestantes sobre o exame citopatológico. Arq Ciênc Saúde. 23(3):62-6. https://doi.org/10.17696/2318- 3691.23.3.2016.288.

Osis, M. J. D., Duarte, G. A. \& Sousa, M. H. (2014). Conhecimento e atitude de usuários do SUS sobre o vírus HPV e as vacinas disponíveis no Brasil. Rev. Saúde Pública. 48(1): 123-33. http://dx.doi.org/10. 1590/S0034.

Rodrigues, A. M. X., Barbosa, M. L. \& Matos, M. D. L. P. (2013). Importância do exame papanicolau no diagnóstico precoce de câncer do colo do útero. Rev Multiprof Saúde Hosp São Marcos. 1(1): 58-65

Sá, M. I., Silva, M. T., Almeida, D., Vieira, B., Lima, T., Conde, C., \& Oliveira, T. (2015). Infeções sexualmente transmissíveis e factores de risco nas adolescentes e jovens: Dados de um Centro de Atendimento a Jovens. Nascer E Crescer-Birth And Growth Medical Journal, $24(2), 64-69$. 
Research, Society and Development, v. 11, n. 1, e21911123312, 2022

(CC BY 4.0) | ISSN 2525-3409 | DOI: http://dx.doi.org/10.33448/rsd-v11i1.23312

Tadeu, V. E. Vacina contra o HPV reduz taxas de câncer de colo de útero em até 87\%. CNN Brasil,2021. https://www.cnnbrasil.com.br/saude/estu do-vacinacontra-o-hpv-reduz-taxas-de-cancer-de-colo-de-utero-em-ate-87.

Toninato, L. G. D., Irie, M. M. T., Consolaro, M. E. L., Teixeira, J. J. V., \& Boer, C. G. (2016). Vaginose bacteriana diagnosticada em exames citológicos de rotina: prevalência e características dos esfregaços de Papanicolaou. Revista Brasileira de Análises Clínicas, 48(2), 165-169. 